\title{
DISSEMINATED ANGIOLYMPHOID HYPERPLASIA WITH EOSINOPHILIA- A THERAPEUTIC CHALLENGE.
}

\author{
Rajesh Rajagopalan
}

1. Consultant Dermatologist. Department of Dermatology, Kovai Medical Centre Hospital, Erode, Tamil Nadu.

\section{CORRESPONDING AUTHOR:}

Dr. Rajesh Rajagopalan.

Consultant Dermatologist,

Kovai Medical Centre Hospital,

68, Perundurai Road Erode-638011,

Tamilnadu.

E-mail: rajrajskin@yahoo.co.in

\begin{abstract}
Angiolymphoid hyperplasia with eosinophilia (ALHE) is a rare disorder characterized by vascular proliferation, eosinophilia in peripheral blood samples and tissue lymphocytosis. The etiology is unknown. There is a female preponderance. It often presents as multiple, grouped red nodules in the periauricular region, scalp line, and neck. Numerous therapeutic approaches have been tried for ALHE including destructive techniques such as electrodesiccation, cryotherapy, surgical excision, radiotherapy or laser (argon, $\mathrm{CO}_{2}$, pulsed dye). We present a disseminated ALHE (DALHE) case which was present for 12 years and aggravated for 12 months that was treated successfully by using oral isotretinoin and pentoxifylline.
\end{abstract}

KEYWORDS: Disseminated angiolymphoid hyperplasia, eosinophilia, pentoxifylline and isotretinoin

INTRODUCTION: Angiolymphoid hyperplasia with eosinophilia is an uncommon, idiopathic, benign, but potentially disfiguring vascular lesion which usually occurs during early and mid adult life ${ }^{1,2}$. Increased incidence in Asian women. It is still uncertain whether this lesion is a true neoplasm or a reactive process. Some cases appear to be associated with trauma, insect bite, HIV infection, pregnancy, and oral contraceptives. It is usually characterized by isolated or grouped papules, plaques, or nodules primarily in the head and neck. Most patients present with lesions in the periauricular region, forehead, or scalp. Rare sites of involvement include the hands, nail beds ${ }^{3}$, shoulders, breasts, penis, oral mucosa, and orbit. Spontaneous regression is common, but persistent or recurrent lesions may require treatment. Several treatments have been reported but surgery is the most efficient one.

CASE REPORT: We report a 42-year-old woman presenting with painful red elevated skin lesions on her face, limbs, and trunk that had been present for 12 years and aggravated for past 1 year. $\mathrm{H} / \mathrm{O}$ of multiple excisional surgeries in the past without any remissions. In fact, she was of opinion that surgery aggravated her condition. General Examination revealed 3 discrete, firm, nontender lymph nodes of size $1 \mathrm{~cm}$ palpable in the posterior triangle of neck. Systemic examination was unremarkable. Dermatological examination revealed erythematous, soft to firm, tender, dome shaped papules and nodules ranging from $2 \mathrm{~mm}$ to $3 \mathrm{~cm}$ over scalp, cheeks, forehead, in and around ears, front of neck, chest, and lower legs. Lab parameters revealed elevated eosinophils $(12 \%)$, ESR $(46 \mathrm{~mm} / 1 \mathrm{hr})$ with normal serum Ig E. HPE was confirmatory 
(vascular hyperplasia, tissue eosinophilia and lymphoid proliferation). We initiated the patient on pentoxifylline (400mgm BID and isotretinoin (20mgm OD). After 12 weeks of therapy, she has achieved complete relief of symptoms and partial relief of signs.

DISCUSSION: Patients with ALHE typically present with an expanding nodule or group of nodules, usually in the vicinity of the ear. Other areas like trunk and extremities are affected in some cases and hence the name DALHE ${ }^{4}$. Regional lymphadenopathy may be observed as in this case.

A CBC count reveals eosinophilia in approximately $20 \%$ of patients as in our case. The clinical presentation of papules around the ears may suggest ALHE, but a biopsy is required to establish the diagnosis.

Treatment is often challenging but is not mandatory. Drugs tried with varied success include steroids (topical/oral/parental/intralesional), pentoxifyllines, indomethacin6, isotretinoin (due to antiangiogenic properties), imiquimod, topical tacrolimus, interferon Alfa $2 \mathrm{~b}$ and intralesional chemotherapeutics (bleomycin, vinblastine, fluorouracil).In our patient, we combined pentoxifylline and indomethacin(given for 12 weeks).After 10 days of commencing therapy, she was pain free and nodules in legs flattened. Facial and neck lesions started decreasing in size and number after 6 weeks. Now she is drug free and on close follow up.

The proposed mechanism of action of pentoxifylline included inhibition of platelet aggregation causing decreased cytokine release (cause for eosinophilia) and vascular proliferation. Dose given in the reported case was $400 \mathrm{mgm}$ TID. The mechanism of action of indomethacin farnesil (prodrug of indomethacin) is not known, but may be related to prostaglandin metabolism. The advised dosage is $400 \mathrm{mgm}$ BID. It is not available in India.

Simple surgical excision is often used, but the lesions tend to recur. Mohs micrographic surgery has been attempted in order to address ALHE through better margin control. Excisions that include the arterial and venous segments at the base of the lesion prove most efficacious. The pulsed-dye laser and carbon dioxide laser have been used with some success. Cryosurgery has also been reported .Conductive hearing loss from obstruction of the auditory canal can occur in severe cases. Diplopia and proptosis was noted in orbital involvement .Lesions can remit over the course of months or years, but they tend to persist or recur.

CONCLUSION: This case is presented to discuss therapeutic challenges faced in the management of widespread recurrent ALHE when the patient refuses to undergo invasive mode of treatment.

\section{REFERENCES:}

1. Mehregan AH, Shapiro L. Angiolymphoid hyperplasia with eosinophilia. Arch Dermatol. Jan 1971; 103(1):50-7.

2. Olsen TG, Helwig EB. Angiolymphoid hyperplasia with eosinophilia. A clinicopathologic study of 116 patients. J Am Acad Dermatol. May 1985; 12(5 Pt 1):781-796.

3. Tsuboi H, Masuzawa M, Katsuoka K. Angiolymphoid hyperplasia with eosinophilia affecting the nail bed and underlying bone. J Dermatol. Jun 2006; 33(6):399-402.

4. Zhang GY, Jiang J, Lin T, Wang QQ Disseminated angiolymphoid hyperplasia with eosinophilia: a case report. Institute of Dermatology, Chinese Academy of Medical Sciences and Peking Union Medical College, People's Republic of China. 
5. Angiolymphoid hyperplasia with eosinophilia may respond to pentoxifylline. Person JR. J Am Acad Dermatol 1994; 31:117-8.

6. Angiolymphoid hyperplasia with eosinophilia :successful treatment with indomethacin farnesil.Nomura K, Sasaki C, Murai T ,et al. Br J Dermatol 1996;134:189-90.

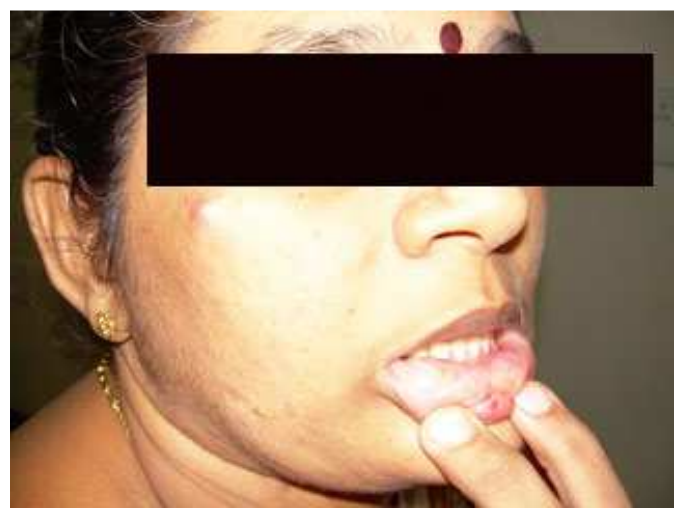

Fig.1 Mucosal lesions

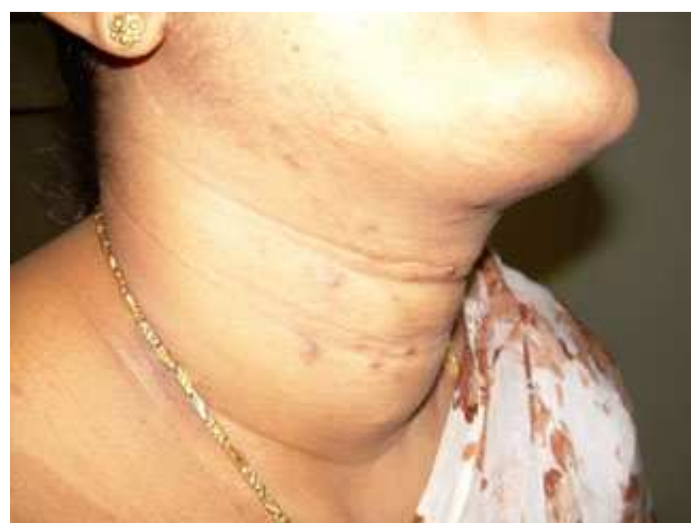

Fig.3 Neck lesions

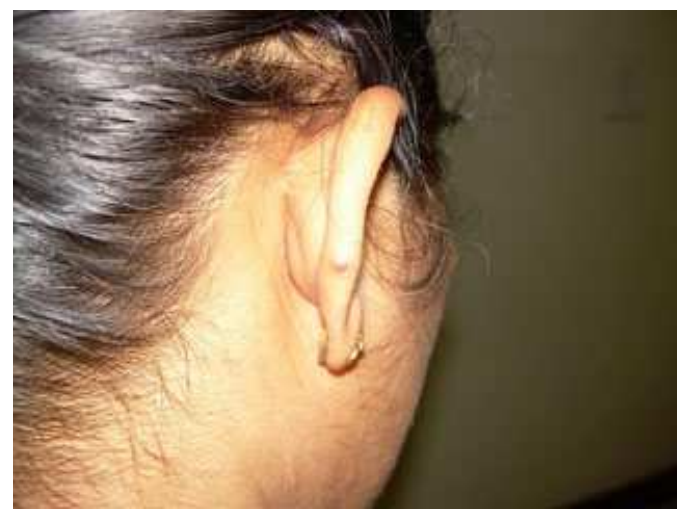

Fig.2 Aural lesions

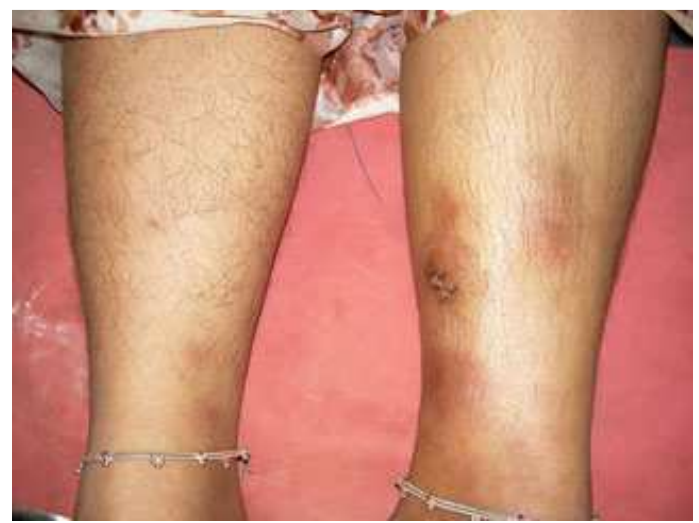

Fig.4 Nodules over shin

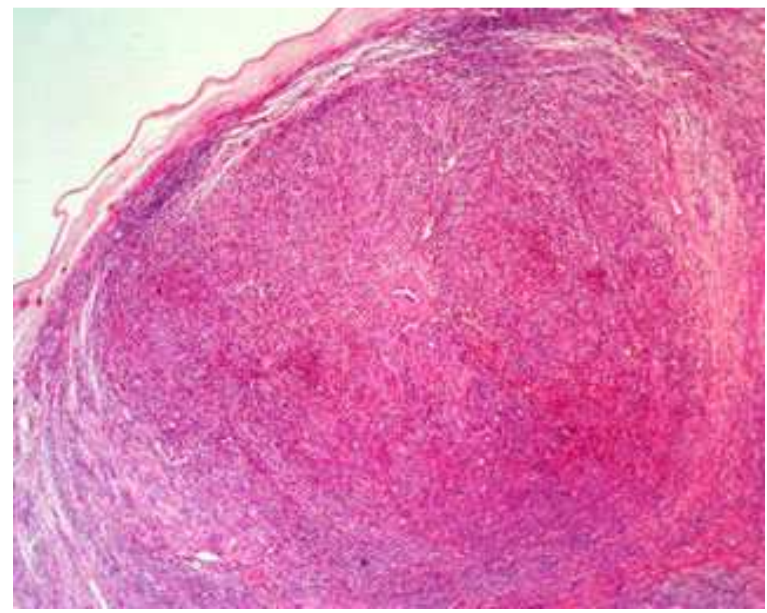

Fig.5 HP (low power magnification- H\&E stain) showing classical features 


\section{CASE REPORT}

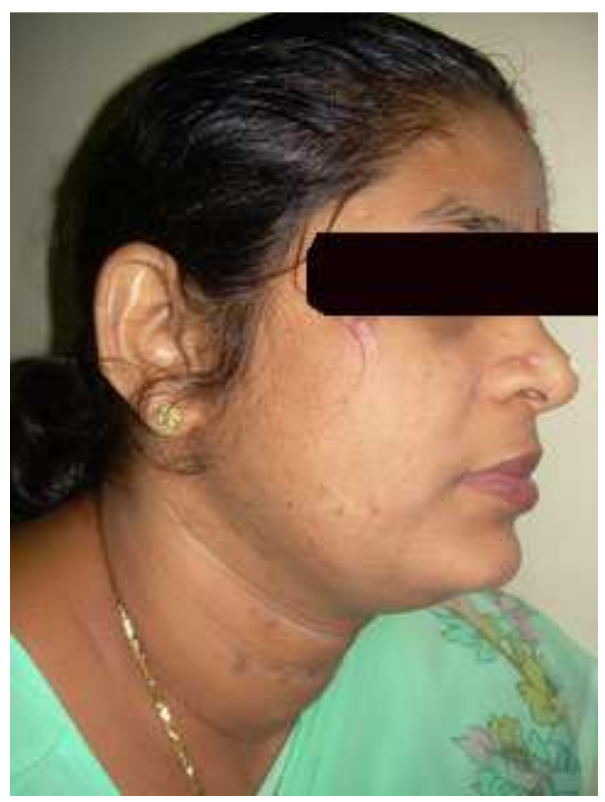

Fig.6 Lesions over head \& neck

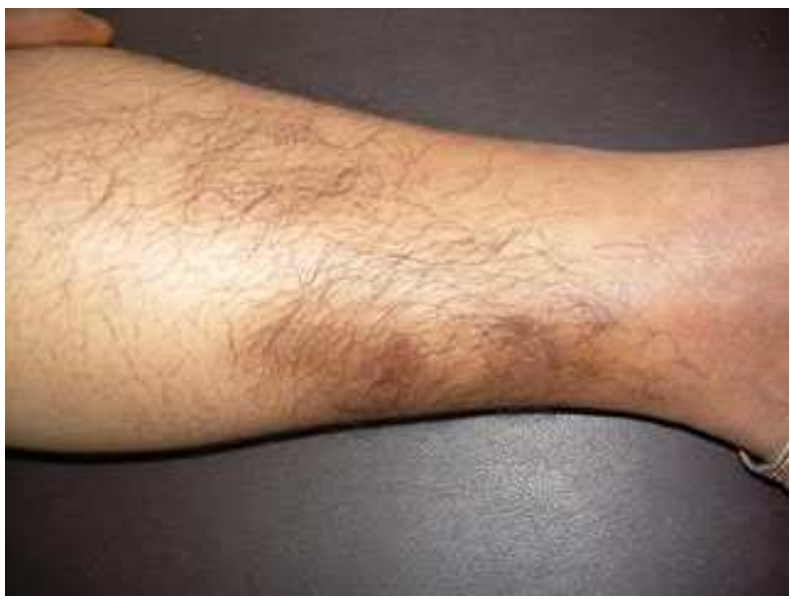

Fig.7 Tender nodules over legs captions for the study 